\title{
Taxonomical Index
}

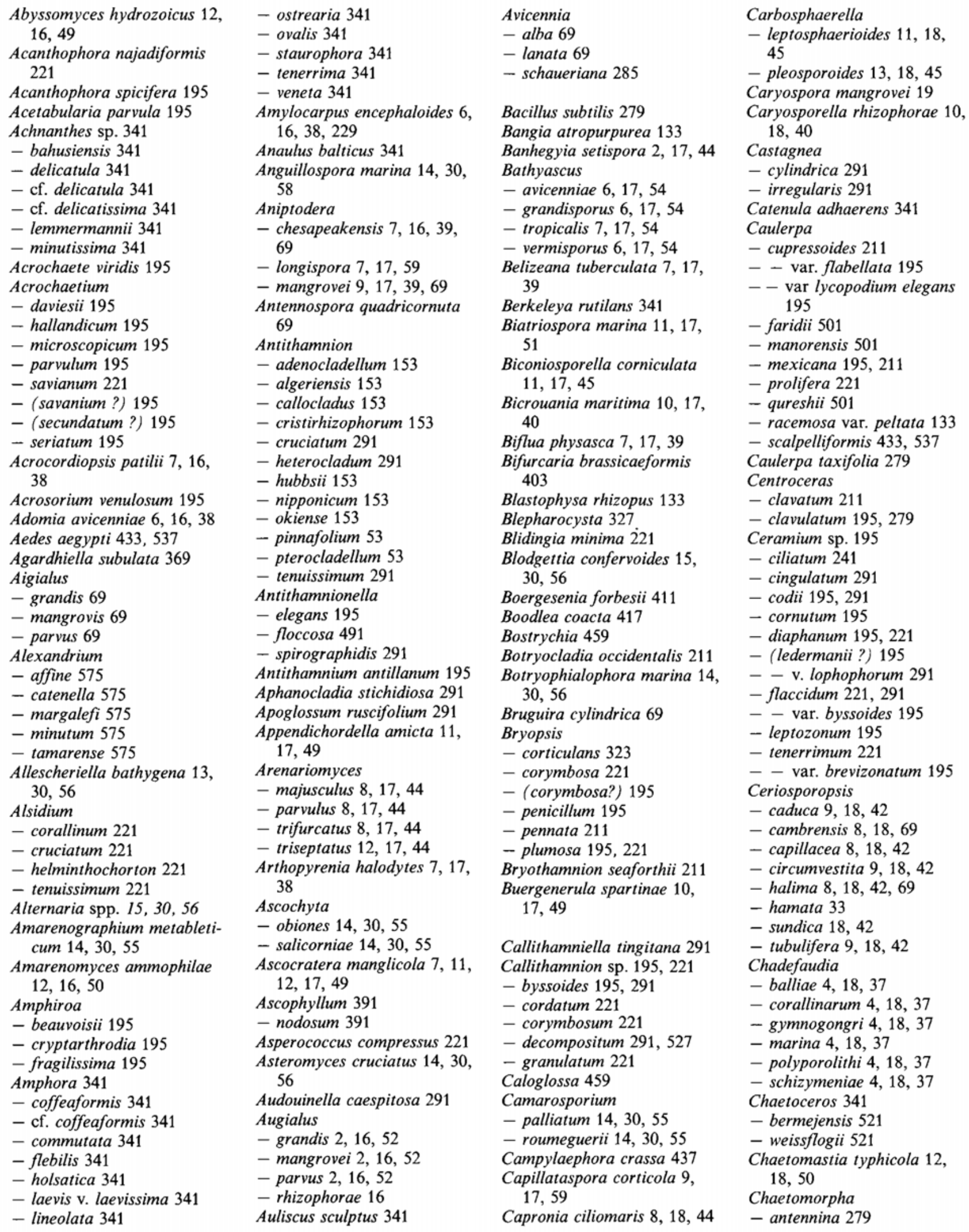


- brachygona 195

- linum 221, 215

- (nodosa?) 1959

Chaetosphaeria chaetosa 12 , 19, 44

Champia parvula 195, 291

Chondria sp. 195

- dasyphylla 195

- macrocarpa 553

- mairei 221

- tenuissima 221

Chondrus crispus 303

Choristocarpus tenellus 541

Cirrenalia sp. 69

- basiminuta 15, 30, 57, 69

- fusca 15, 30, 57

- macrocephala 15, 30, 57. 69

- pseudomacrocephala 15 , 30,57

- pygmea $15,30,57,69$

- tropicalis 5, 30, 57

Cladophora

- albida 291

- dalmatica 221, 241

- fascicularis 279

- glomerata 221

- hutchinsiae 221

- laetevirens 221

- prolifera 195, 221

- ruchingeri 221

- rupestris 221

- sericea 221

- (vagabunda?) 195

Cladosporium algarum 15, 31, 56

Clavariopsis bulbosa 69

Clavatospora

- bulbosa 15, 31, 58

- stellatacula 14, 31, 56

Cocconeis 341

- cf. diminuta 341

- peltoides 341

- cf. peltoides 341

- placentula 341

- scutellum 341

- stauroneiformis 341

Codium

- decorticatum 221

- dwarkense 501

- effusum 241

- flabellatum 501

- isthmocladum 195

- taylori 195

- vermilara 241

Colpomenia sinuosa 77, 195, 241

Compsothamnion thuyoides 291

Coniothyrium obiones 14,31 , 55

Corollospora sp. 2, 19, 46

- angusta 2, 19, 46

- armoricana 3, 19, 46

- cinnamomea 2, 19, 46

- colossa 2, 19, 48

- filiformis 3, 19, 47

- fusca 2, 19, 48

- gracilis 2, 19, 46

- intermedia 2, 19, 46

- laceraa 3, 19, 47

- luteola 3, 19, 47

- maritima 2, 19, 46
- novofusca 2, 19, 34, 48

- pseudopulchella 3, 19, 47

- pulchella 3, 19, 47

- quinqueseptata 3, 19, 47

Corallina

- (cubensis ?) 195

- granifera 195

- officinalis 211, 221, 241

- rubens 429

- subulata 211

Corollospora maritima 69

Coronopapilla

- avellina 19

- mangrovei 11, 19, 40

Cottoniella filamentosa 195

_ _ var. fusiformis 195

Cremasteria cymatilis 33

Crinigera maritima 5, 20, 44, 229

Crouania

- attenuata 291

- franciscii 195

Cryptonemia

- crenulata 211

- seminervis 195

Cumulospora

- mangrovei 9, 20, 43

- marina 16, 31, 57

Cyclotella

- atomus 341

- caspia 341

Cylindrodendrum album var. paralion 33

Cylindrotheca closterium 341

Cymodocea nodosa 319

Cystoseira spp. 541

- barbata 221, 541

- compressa 221, 241

- corniculata 241

- discors 195

- humilis 195

- mauritanica 195

- spicata 241

Cytospora rhizophorae 13, 31, 55

Dactylospora haliotrepha 2, 20,39, 69

Dasya sp. 195

- baillouviana 195

- (caraibica?) 195

- hutchinsiae 291

Delesseria sanguinea 429

Dendryphiella

- arenaria 15, 31, 56

- salina 15, 31, 56

Derbesia sp. 195

- tenuissima 133

Dermonema abbottiae 211

Diatoma tenue 341

Dictyopteris

- delicatula 195

- membranacea 195, 241

Dictyosporium pelagicum 15 , $31,56,69$

Dictyota spp. 195

- cervicornis 195

- dichotoma 195, 221, 241, 291, 425, 433, 537

- - var. intricata 221

- dumosa 425

- hauckiana 425

- cf. indica 221, 425
- linearis 221

- maxima 425

Didymella

- avicenniae 7, 20, 39

- fucicola 5, 20, 38

- gloeopeltidis 5, 20, 38

- magnei 5, 20, 38

Didiymosphaeria

- enalia 69

- lignomaris 10, 20, 40

Digitatispora

- lignicola 13, 29, 55

- marina 13, 29, 55

Dimerogramma minor 341

Dinemasporium marinum 13, 31, 55

Diplodia oraemaris 14, 31, 55

Diploneis finnica 341

Ditylum brightwellii 505

Dohrniella antillarum 195

Dryosphaera navigans 8,20 , 44, 229

Dudresnaya sp. 195

Ecklonia

- kurome 387

- radiata 261

Ectocarpus siliculosus 241

- - var. confervoides 221

Eiona tunicata 6, 21, 38, 229

Emiliania huxleyi 589

Enterobacter aerogenes 279

Enteromorpha spp. 241

- - ssp. linziformis 221

- clathrata 195

- compressa 221, 279

- flexuosa 221, 365

- intestinalis 221

- multiramosa 291

- prolifera 221

- ramulosa 195

Entocladia sp. 195

- major 195

Entomoneis paludosa v. duplex 341

Epicoccum spp. 16, 31, 57

Erythroglossum sandrianum 291

Erythrotrichia carnea 291

Erythrotrychia

- boryana 195

- carnea 195

- simplex 195

Escherichia coli 279

Etheirophora

- bijubata 8, 21, 44

- blepharospora 8, 21, 44

- unijubata 8, 21, 44

Eupogodon

- pilosus 133

- planus 291

Exserohilum spp. 15, 31, 56

Falkenbergia

- hillebrandii 195, 541

- rufalanosa 291, 541

Feldmannia indica 195

Feldmannophycus rayssiae 221

Fosliella farinosa 195, 221, 291

Fragilaria vaucheriae 341

Fucus 391
- vesiculosus 447

- virsoides 241

Gastroclonium clavatum 221

Gelidiopsis gracilis 211

Gelidium

- crinale 195, 221

- pusillum 195, 221, 241, 279

- spathulatum 221

Giffordia mitchelliae 291

Gigartina

- acicularis 195, 221, 279, 429

- radula 403

Giraudia sphacelarioides 291

Gloeosporidina cecidii 13, 31, 55

Gloiocladia furcata 291

Gnomonia salina 9, 21, 43

Gomphonemopsis exigua 341

Gracilaria sp. 383

- blodgettii 139

- bursa-pastoris 221

- cearensis 211

- cervicornis 211

- conferta 161

- cornea 211

- corticata 107, 279

- domigensis 211

- dura 221

- ferox 211

- foliifera 107

- longa 221

- sjoestedtii 211

- tikvahiae 139

- verruscosa 221

Grateloupia

- dichotoma 429

- filicina 221, 279

- lithophila 279

Griffithsia spp. 195

- cf. arachnoidea 221

- opuntioides 221

- schousboei 221

Groenhiella bivestia 10, 21, 45

Gymnascella littoralis 6, 21, 38

Gymnogongrus furcellatus 145

\section{Haligena}

- elaterophora 12, 21, 42

- salina 9, 21, 42

Halimeda

- copiosa 291

- cryptica 291

- discoidea 291

- dixoidea 291

- floresia 195

- gracilis 291

- incrassata 291

- lacrimosa 291

- monile 291

- opuntia 291

- simulans 291

- tuna 291

Haliptilon subulatum 195

Halochloroccum 459

Halocyphina villosa 13, 30, 55

Halodule wrightii 265 
Halographis runica 2, 21, 38

Haloguignardia

- cystoseirae 4, 21, 37

- decidua 4, 21, 37

- irritans 4, 21, 37

- oceanica 4, 21, 37

- tumefaciens 4, 21, 37

Halonectria milfordensis 6 , $13,21,31,38,55$

Halopteris scoparia 241

Halosarpheia sp. 9, 22, 59

- abonnis 9, 21, 43

- bentotensis $12,21,43$

- cincinnatula 12, 21, 43, 69

- fibrosa 9, 21, 43

- indica 33

- lotica 69

- marina 9, 21, 43, 69

- minuta 69

- ratnagiriensis $9,21,43,69$

- retorquens $9,21,43$

- spartinae 12, 21, 43

- trullifera 9, 22, 43

- unicaudata 12, 22, 43

- viscidula 12, 22, 43

- viscosa 9, 22, 43, 69

Halosphaeria

- appendiculata 10, 22, 41, 69,229

- cucullata 8, 12, 22, 39

- quadricornuta 8, 22, 41

- salina $8,22,41$

- viscosa 22

Halosphaeriopsis mediosetigera $10,22,45$

Halosyphina villosa 69

Halotthia posidoniae 10, 22, 40

Halymenia

- actinophysa 195

- floresia 195

Hantzschia marina 341

Heleococcum japonense 7, 22, 39

Helicascus

- nypae 311

- kanloanus 10, 22, 40, 69, 311

Helminthocladia

- agardhiana 527

- australis 81

- calvadosii 81

- densa 81

- dotyi 81

- nizamuddinii 81

Herposiphonia

- secunda 195

- - var. tenella 195, 221

Heterosigma akashiwo 113

Heterosiphonia crispella 195

Hildenbrandia 201

Humicola alopallonella 15 , $31,56,69$

Hydronectria

- tethys 7, 22, 39, 69

- - var. glabra 7, 22, 39

Hydropuntia dentata 195

Hypnea

- arbuscula 195

- cervicornis 195, 211, 221

- musciformis 195, 211, 221, 279

- valentiae 279
Hypoglossum hypoglossoides 291

Hypophloeda rhizospora 11 , 22,59

Hypoxylon oceanicum 6, 22, 40,69

Iyengaria stellata 77

Jania

- adhaerens 195

- capillacea 195

- longifurca 527

- rubens 195, 241

- (verrucosa?) 195

Katodinium rotundatum 113

Kirschsteiniothelia maritima $10,22,40$

Kohlmeyeriella tubulata 6, 23, 38

Koralionastes

- angustus 3, 23, 51

- ellipticus 3, 23, 51

- giganteus 3, 23, 51

- ovalis 3, 23, 51

- violaceus 3, 23, 51

Kuckuckia spinosa 541

Laboulbenia marina 7, 23, 39

Laetinaevia marina 2, 23, 37

Laguncularia racemosa 279

Laingia pacifica 553

Landsburgia quercifolia 455

Lanspora coronata 6, 23, 44

Laurencia

- nipponica 553

- obtusa 211, 241, 553

- paniculata 195

- (papillosa?) 195

- papillosa 553

- perforata 195

- pinnatifida 221

- undulata 553

Lautitia danica 5, 23, 38

Lautospora gigantea 13, 23, 52

Leptosphaeria

- australiensis 11, 23, 49

- avicenniae 12, 23, 49

- oraemaris $11,23,50$

- pelagica 11, 12, 23, 49

- peruviana 11, 23, 50

Leptosphaeria sp. 229

Levringia atlantica 195

Lignincola

- laevis 7, 23, 38, 69

- longirostris 7, 23, 38, 69

- tropica 7, 23, 38

Lindra

- crassa 3, 23, 53

- hawaiiensis 3, 23, 53

- inflata 4, 23, 53

- marinera 4, 23, 53

- obtusa 3, 24, 53

- thalassiae 4, 24, 53

- - var. crassa 23

Lineolata rhizophorea 10, 24, 40

Linocarpon

- appendiculatum 4, 24, 53

- nypae 4, 24, 53

- cfr. pandani $4,24,53$
Lissodinium 327

Lobophora variegata 195

Lomentaria

- articulata 221

- chylocladiella 291

- compressa 221

- subdichotoma 221

Lophiostoma mangrovei 2 , 24,49

Lulworthia

- calcicola 3, 24, 54

- crassa 3, 24, 54, 69

- curalii 3, 24, 54

- fucicola 3, 24, 54, 69

- grandispora 3, 24, 54, 69

- kniepii 3, 24, 54

- var. curalii 24

- medusa 69

- lignoarenaria 3, 24, 54, 69

- lindroidea 3, 24, 54

- uniseptata 3, 24, 54, 69

- spp. 3, 24, 69

Luttrellia estuarina 10, 24, 51

Macrocystis pyrifera 465

Macrophoma spp. 14, 31, 55

Manglicola guatemalensis 6 , 24,40

Marinosphaera mangrovei 11 , 24, 49

Marinospora

- calyptrata 10, 24, 42

- longissima 10, 25, 42

Marisolaris ansata 10, 25, 45

Massarina

- acrostichi 7, 25, 49

- cystophorae 5, 25, 42

- lacertensis 7, 25, 49

- thalassiae 11, 12, 25, 49

- velatospora 12, 25, 49,69

Massariosphaeria typhicola 33

Mastodia tessellata 5, 25, 37

Melanotaenium ruppiae 13 , 30

Melobesia membrancea 195

Micromonas pusilla 113

Moana turbinulata 6, 25, 38

Monodictys pelagica 15, 31, 57, 69

Mycaureola dilseae 13, 30, 55

Mycosphaerella

- apophlaeae 5, 25, 38

- ascophylli 5, 25, 38

- pneumatophorae 7, 25, 38, 69

- salicorniae 7, 25, 38

- staticicola 7, 25, 38

- suaedae-australis 7, 25

Myriogramme carnea 291

Myrionema sp. 195

- magnusii 291

Nais

- glitra 7, 25, 39, 69

- inornata 7, 25, 39, 69

Nautosphaeria cristaminuta 6 , 25,44

Navicula

- abscondita 341

- amphipleuroides 341

- arenaria 341

- atlantica 341
- cf. biskanteri 341

- cincta 341

- clamans 341

- cryptolyra 341

- digitoradiata 341

- cf. flanatica 341

- forcipata 341

- gregaria 341

- halophila 341

- heterovalvata 341

- humerosa 341

- cf. jaernefeltii 341

- lanceolata 341

- cf. perminuta 341

- phyllepta 341

- pygmaea 341

- rhynchocephala 341

- salinarum 341

- salinicola 341

- subapiculata 341

- subinflata 341

- wuestii 341

Nectriella laminariae 5, 25, 38

Nemacystus

- erythraeus (var. hispanicus?) 195

- flexuosus 291

- novae-zelandiae 133

Nereiospora

- comata 13, 26, 45

- cristata 12, 26, 45

Nia vibrissa $13,30,55$

Nimbospora

- bipolaris 8, 26, 45

- effusa 8, 26, 45

- octonae 8, 26, 45

Nithophyllum

- micropunctatum 291

- punctatum 195

Nitzschia

- aurariae 341

- dissipata 341

- frustulum 341

- cf. linearis 341

- littorea 341

- paleacea 341

- cf. spathula 341

- thermaloides 341

Oceanitis scuticella 7, 26, 43

Ocostaspora apilongissima $10,26,41$

Odontella regia 505

Ondiniella torquata $9,26,42$

Opephora 341

- olsenii 341

Ophiobolus australiensis 33

Ophiodeira monosemeia 8, 26, 43

Orbimyces spectabilis 15,31 , 58

Orcadia ascophylli 5, 26, 49

Padina

- pavonica 241

- tetrastromatica 195

- vickersiae 195

Papulospora halima 33

Paraliomyces lentiferus 8, 26, 39

Passeriniella

- incarcerata 26,59 
- obiones 11, 26, 40

- savoryellopsis 11, 26, 40, 69

Payosphaeria minuta 6, 26, 59,69

Pentapharsodium dalei 91

Periconia

- abyssa 14, 31, 56

- prolifica 14, 32, 56, 69

Peridinium

- quinquecorne 123

Petalonia fascia 221

Peyssonnelia 201

Phaeosphaeria

- albopunctata 33

- gessneri 11, 26, 50

- halima 12, 27, 50

- macrosporidium 11, 27, 50

- neomaritima 12, 27, 50

- spartinae $11,27,50$

- spartinaecola 11, 27, 50

- typharum 11, 27, 50

Pharcidia

- laminariicola 5, 27, 50

- rhachiana 5, 27, 38

Phialophorophoma litoralis $14,32,55$

Phoma

- laminariae 14, 32, 55

- marina 33

- suaedae $14,32,55$

- spp. 14, 32, 55

Phomatospora acrostichi 6, 27, 38

Phycodrys rubens 375

Phycomelaina laminariae 5, 27, 39

Pinnularia

- ambigua 341

- rectangulata 341

Plagiogramma staurophorum 341

Platysiphonia

- caribaea 527

- miniata 527

Pleonosporium borreri 195

Pleospora

- avicennia 13, 27, 52

- gaudefroyi $13,27,52$

- gracilaria 5, 27, 59

- pelagica 13, 27, 52

- pelvetiae 5, 27, 52

- spartinae 13, 14, 27, 32, 52,55

- triglochinicola 13, 28, 52

Pleurosigma

- angulatum 561

- rhombeum 561

- stidolphii 561

Plocamium cartilagineum 195, 291

Pneophyllum

- lejolisii 195

- confervicola f. minutula 195

Podospora inquinata 33

Polysiphonia sp. 195, 391

- cf. elongata 291

- gorgoniae 195

- lanosa 391

Polystigma apophlaeae 5, 28, 37

Pontogeneia
- calospora $6,28,51$

- codiicola 5, 28, 51

- cubensis 5, 28, 51

- enormis 5, 28

- erikae 5, 28, 51

- padinae 6, 28, 51

- valoniopsidis 5, 28

Pontoporeia biturbinata 10 , 28,40

Porphyra

- capensis 403

- leucosticta 221, 241

- linearis 221

- umbilicalis 221

Posidonia oceanica 265, 319

Predaea huismanii 527

Pronectria laminariae 36

Proteus vulgaris 279

Pseudochlorodesmis furcellata 195

Pseudodictyon inflatum 195

Pseudoscinaia 513

Pseudostaurosira 341

Pterocladia capillacea 177, 187, 241

Pterothamnion

- crispum 291

- plumula 221, 291

Ptilothamnion pluma 291

Pyrenocollema pelvetiae 5 , 28, 38

Quintaria lignatilis 11, 28, 35, 49

Radicilingua

- reptans 221

- thysanorhizans 221

Remispora

- crispa 9, 28, 41

- galerita 9, 28, 41

- maritima 9, 28, 41

- pilleata 9, 28, 41

- quadriremis $8,28,41$

- spinibarbata 9, 28, 41

- stellata 9, 28, 41

Rhabdospora avicenniae 13 , 32,55

Rhizophila marina 6, 28, 38, 69

Rhizophora

- apiculata 69, 537

- mangle 285

- mucronata 69

Rhodophyllis divaricata 195 , 291

Rhodymenia pseudopalmata 195, 221

Rhoicospenia abbreviatia 341

Rissoella verruculosa 527

Robillarda rhizophorae 14, 32,55

Rosellinia sp. 69

Rosenvingea sanctae-crucis 195

Ruppia maritima 265

Rytiphloea tinctoria 195, 221

Sahlingia tinctoria 195

Salmonella

- paratyphi 2B 279

- typhi 279
Salsuginea 311

- ramicola 311

Sargassum

- filipendula 195

- ramifolium 195

- vulgare 195

- wightii 279

Savoryella

- lignicolla 11, 28, 40, 69

- paucispora 11, 28, 40, 69

Scinaia

- boergensii 513

- cottonii 513

- flabellata 513

- hormoides 513

- howensis 513

- japonica 513

- moniliformis 513

- okiensis 513

- pseudo-moniliformis 513

- tokidae 513

Scrippsiella

- arenicola 167

- caponii 167

- crystallina 91

- hexapraecingula 167

- lachcrymosa 91

- mexicana 167

- precaria 167

- spinifera 167

- saladense 167

- subsalsa 167

- tinctoria 167

- trifida 91

- trochoidea 91, 167

Scytosiphon lomentaria 221, 241

Seirospora

- interrupta 291

- sphaerospora 291

Septoria

- ascophylli 33

- thalassica 33

Serratia marcescens 279

Sigmoidea

- luteola 14, 32, 58

- marina 14, 32, 58

Soliera

- filiformis 221

- tenera 195

Sonneratia alba 69

Spathulaspora

- adelpha 4, 28, 37

- antarctica 4, 28, 37

- calva 4, 29, 37

- lanata 4, 29, 37

- phycophila 4, 29, 37

Spatoglossum schroederi 195

Spermothamnion sp. 195

- flabellatum 527

- (investiens?) 195

- johannis 291

- macromeres 195

- (repens?) 195

Sphacelaria

- cirrosa 291

- rigidula 195

- sp. 195

- tribuloides 195

Sphaeria incarcerata 26, 59

Sphaerulina

- albispiculata 33

- oraemaris 11, 29, 49, 69
Sphondylothamnium multifidum 291

Splachnidium rugosum 403

Splanchnonema britzelmayriana 34

Sporidesmium salinum 14, 32, 58

Sporochnus bolleanus 195

Spyrida

- clavata 195

- filamentosa 195, 221, 359

- hypnoides var. typica 195

Stagonospora

- haliclysta 14, 32, 58

- sp. 14, 32, 55

Staphylococcus aureus 279

Stauroneis

- salina 341

- tackei 341

Stemphylium

- gracilariae 16, 32, 59

- triglochinicola 16, 32, 57

Stictyosiphon

- adriaticus 221

- soriferus 221

Striatella delicatula 341

Stylonema

- alsidii 195, 221, 291

- cornucervi 221, 291

Swampomyces armeniacus 7 , 29,38

Syringodium filiforma 265

Taenioma sp. 195

Thalassia

- hemprichii 271

- testudinum 265

Thalassogena sphaerica 6, 29, 38

Thalassosacus

- cystoseirae 5, 29, 39

- lessoniae 5, 29, 39

- tregoubovii 5, 29

Torpedospora

- ambispinosa 12, 29, 44

- radiata $12,29,44$

Trailia ascophylli 5, 29, 49

Trematosphaeria

- mangrovei 11, 29, 50

- striatispora 12, 29, 50

Trichocladium

- achrasporum 15, 32, 56, 69

- constrictum 15, 32, 56

- lignincola 15, 32, 56

Trichomaris invadens 12,29 , 42

Tropidoneis lepidoptera 341

Tubercularia pulverulenta 13, 32,56

Turgidosculum ulvae 5, 29, 37

Ulva

- curvata 221

- lactuca 211, 279, 475, 483

- olivascens 221

- rigida 221, 241, 251, 403

- rotundata 221

- scandinavica 221

Ulvella lens 195

Varicosporina

- prolifera 14, 32, 58 
- ramulosa 14, 32, 58

Verrucaria cribbii $6,29,37$

Verruculina enalia 10, 29, 40
Wettsteinia marina 11, 29, 50

Wrangelia penicillata 291

Xylaria psamathos 34
Zalerion

- maritimum 15, 33, 57, 69

- varium 15, 33, 57

Zea mays 469

Zopfiella

- latipes 6, 29, 40
- marina 6, 29, 40

Zostera

- capricorni 319

- noltii 319

- marina 319 


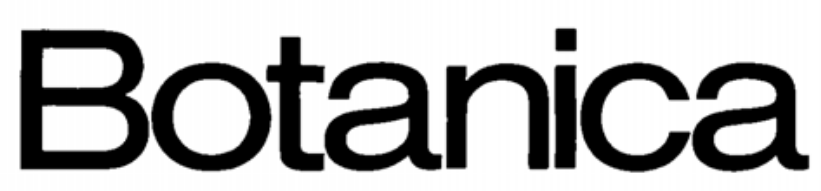

\section{Marina voume 34, 1991}

Editors M. A. Borowitzka, Australia - J. Cabioch, France - T. Hori, Japan - E. B. G. Jones, Great Britain - J. Kohlmeyer, U.S.A.

D. G. Müller, F. R. of Germany · M. Neushul, U.S.A. · R. E. Norris, South Africa ·

E. C. de Oliveira, Brazil - M.-R. Plante-Cuny, France - M. A. Ragan, Canada - P. C. Silva, U.S.A. C C. K. Tseng, China - A. I. Usov, U.S.S.R. - I. Wallentinus, Sweden

Editor-in-Chief

G. T. Boalch, Great Britain 
Plymouth PL1 2PB

Great Britain

Telephone (0752) 222772

$\begin{array}{ll}\text { Telefax } & \text { (0752) } 226865\end{array}$

\section{Editorial Office}

Botanica Marina

Genthiner Str. 13

D-1000 Berlin 30

F. R. of Germany

Telephone: (030) 260 05-133

$\begin{array}{lll}\text { Telefax: } & \text { (030) } 26005-251\end{array}$

Telex: 184027

\section{Attention before copying!}

Do you photocopy articles from this periodical? If so, have you made certain that you are not violating the legal copyright regulations and making yourself liable to prosecution?

According to copyright law, it is permissible to make a few copies of individual articles for personal use only. Reproduction of articles for commercial use by an industrial enterprise is subject to charge. Detailed information can be obtained free of charge from the VG Wissenschaft GmbH, "Copyright, Großer Hirschgraben 17-21, D-6000 Frankfurt/Main, this company being responsible for collection of copying fees.

\section{Copying in the U.S.A.}

Authorization to photocopy items for internal or personal use, or the internal or personal use of specific clients, is granted by Walter de Gruyter \& Co. - Berlin. New York for libraries and other users registered with the Copyright Clearance Center (CCC) Transactional Reporting Service, provided that the base fee of $\$ 02.00$ per copy is paid directly to CCC, 21 Congress St., Salem, MA 01970.

0006-8055/90/\$02.00

Title No. 3109006767

ISSN 0006-8055

Copyright (C) 1991 by Verlag Walter de Gruyter \& Co.

Typesetting and Printing Arthur Collignon GmbH, Berlin

Binding Buchbinderei Spiller, Berlin

Editorial Assistant and Technical Coordinator Asta Wonneberger

Printed in Germany

All rights reserved, including those of translations into foreign languages. No part of this journal may be reproduced in any form - by photoprint, microfilm or any other means - nor transmitted nor translated into a machine language without written permission from the publisher.

The quotation of registered names, trade names, trade marks, etc. in this journal does not imply, even in the absence of a specific statement, that such names are exempt from laws and regulations protecting trade marks, etc. and therefore free for general use.

Walter de Gruyter \& Co., Genthiner Straße 13, D-1000 Berlin 30, F.R. of Germany Tel. (030) 260 05-133, Telefax (030) 260 05-251, Telex 184027

Walter de Gruyter, Inc., 200 Saw Mill River Road, Hawthorne, N.Y. 10532, U.S.A.

Tel. (914) 747-0110, Telefax (914) 747-1326, Telex 646677

\section{Walter de Gruyter · Berlin · New York}

